\title{
Importância do treinamento e desenvolvimento nos serviços de saúde
}

\author{
Ana Isabel Passos Leandro \\ Graduada em Administração Hospitalar \\ Elisângela de Sousa Branco \\ Prof $^{\mathrm{a}} \mathrm{Dr}^{\mathrm{a}}$ da Autarquia de Ensino Superior de Garanhuns - AESGA e \\ da Autarquia de Ensino Superior de Arcoverde - AESA \\ brancoesb@hotmail.com
}

\begin{abstract}
RESUMO: O treinamento é um dos meios de desenvolver competências das pessoas para se tornarem mais produtivas, criativas e inovadoras, a fim de corresponderem aos objetivos organizacionais. O objetivo da presente pesquisa é mostrar a necessidade de treinamento e desenvolvimento nos serviços de saúde, destacando a importância da qualidade na assistência das redes hospitalares, salientando a educação continuada. A administração das instituições de saúde também se responsabiliza por seu desempenho, coordenando e planejando desde a implantação de rotinas, como os equipamentos necessários ao seu funcionamento até a área de Recursos Humanos. A metodologia utilizada foi através de pesquisa bibliográfica por meio de artigos localizados na base de dados, busca manual em livros e jornais científicos. Diante da análise qualitativa dos artigos ficou diagnosticada a necessidade de treinar e desenvolver habilidades e competências dos funcionários para que atinjam as metas institucionais que tem como foco a excelência no atendimento. Concluiu-se que se faz necessário o desenvolvimento dos Recursos Humanos, pois prestar bons serviços depende da qualidade do desempenho dos funcionários. E ainda os hospitais precisam enfrentar o desafio de oferecer conhecimento e oportunizar crescimento para sua equipe.
\end{abstract}

PALAVRAS-CHAVE: Treinamento; Desenvolvimento das Habilidades; Desempenho Profissional.

\section{Importance of training and development in health services}

ABSTRACT: The training is one of the ways to develop abilities of the people to become more productive, creative and innovative, in order to correspond to the organizacionais objectives. The general objective of the present research is to show to the necessity of training and development in the health services, detaching the importance of the quality in the assistance of the hospital nets, pointing out the continued education. The administration of the health institutions also makes responsible for its performance, co-ordinating and planning since the implantation of routines, as the necessary equipment to its functioning until the area of human resources. The used methodology was through bibliographical research by means of articles located in the searchs manual in scientific books and periodicals. Ahead of the qualitative analysis of articles she was diagnosised the necessity to train and to develop abilities and abilities of the employees so that they reach the objectives of the organization that has as focus the excellency in the attendance. It was concluded that the development of Human resources becomes necessary, therefore to give good services depends on the quality of the performance of the employees. E still the hospitals need to face the challenge to offer knowledge and to oportunizar growth for its team.

KEYWORDS: Training; Development of the Abilities; Professional Performance 


\section{INTRODUÇÃO}

A proposta desta pesquisa baseou-se em um novo perfil de gerente envolvido com treinamento e desenvolvimento mostrando como as instituições de saúde podem utilizar essas ferramentas para oferecer uma assistência de qualidade.

Os hospitais, desde sua origem, estiveram voltados para a prestação de serviços de saúde sem se importar com o gerenciamento, mas, em meados dos anos oitenta essa realidade começou a mudar.

As organizações vêm passando por uma reestruturação funcional. Quando a organização sofre mudanças, exige que sua equipe passe por um processo de desenvolvimento de conhecimentos e competências.

O treinamento pode ser um dos meios de desenvolver competências das pessoas para se tornarem mais produtivas, criativas e inovadoras, a fim de corresponderem aos objetivos organizacionais.

A administração pode ser compreendida sob um foco de planos e ações capazes de oportunizar à instituição sua continuidade. Ressalta-se ainda que, de acordo com Maximiano (2000), a administração pode ser compreendida como um processo de tomar decisões e realização de ações para alcançar o seu objetivo.

As instituições de saúde têm como objetivo a excelência no atendimento, mas, não se preocupam em investir em treinamento de seus funcionários, priorizam aquisições de maquinários e aparelhos tecnológicos e, desta forma, o funcionário acaba por investir no autodesenvolvimento, já que, a instituição está alheia a essa necessidade, focando seus objetivos pessoais e deixando de lado as perspectivas da instituição. Fazer com que uma organização realmente se preocupe com o cliente é um desafio muito grande. Não é barato ou fácil e não se consegue sem muito esforço. Não é uma solução fácil e simples (LAS CASAS, 2008).

A administração das instituições de saúde também se responsabiliza por seu desempenho, coordenando e planejando desde a implantação de rotinas, como os equipamentos necessários ao seu funcionamento até a área de Recursos Humanos.

Portanto, o objetivo da presente pesquisa é mostrar a necessidade de treinamento e desenvolvimento como um instrumento para alcançar a excelência na qualidade dos serviços prestados nas organizações de saúde, salientando a importância da educação continuada.

\section{METODOLOGIA}

Este estudo constituiu-se de pesquisa bibliográfica, localizados nas bases de dados: Medline, Lilacs, Scielo, Capes, realizada no período de 2000 a 2009. A seleção de fontes para o levantamento das publicações foi realizada por meio de busca em artigos localizados na base de dados, busca manual em livros e jornais científicos. Foram inclusos artigos que relacionavam o treinamento e desenvolvimento como uma ferramenta utilizada pelos hospitais para garantir uma assistência de qualidade. Houve combinação dos descritores como: treinamento; desenvolvimento e desempenho profissional. Para atender a estes requisitos, o trabalho abrangeu os subtemas: treinamento e desenvolvimento, descrevendo seu conceito, bem como suas funções e objetivos e no segundo capitulo foram relatados os diagnósticos da necessidade de treinamento e desenvolvimento.

\section{TREINAMENTO E DESENVOLVIMENTO}

\subsection{Conceito}

Na década de 90 , devido ao surgimento da era da informação, as organizações tiveram que passar por uma mudança funcional para poderem acompanhar tais variações. Investiu-se muito na qualidade total, e com isso as organizações chegaram a um mesmo patamar. Mas para se diferenciarem passaram a investir no conhecimento e desenvolvimento (BRANDÃO; GUIMARÃES, 2001).

O gestor comprometido com qualidade na assistência pode ter êxito em seus objetivos administrativos se as pessoas que trabalham para ele forem capazes de satisfazer os padrões funcionais. O administrador precisa oferecer às pessoas uma compreensão clara das responsabilidades inerentes à função, depois, fazse necessário dar-Ihes a autoridade para assumir essas responsabilidades, estabelecendo e transmitindo os padrões de excelência que dizem às pessoas como desempenhar a sua função. Para atender a esses padrões, segundo Pio (2006) as pessoas precisam ser treinadas. 
O treinamento de acordo com Lacerda; Abbad (2003) é apenas uma das intervenções que afetam o desempenho no trabalho. É, portanto, somente uma das tentativas formais de garantir a aquisição de novos conhecimentos, habilidades e maior compreensão da tarefa, não afetando, necessariamente, condições organizacionais (materiais e sociais) relacionadas ao desempenho.

Gil (2006) refere-se como treinamento ao conjunto de experiências de aprendizagem centradas na posição atual da organização. Trata-se, portanto, de um processo educacional de curto prazo e que envolve todas as ações que visam deliberadamente ampliar a capacidade das pessoas para desempenhar melhor as atividades relacionadas ao cargo que ocupam na empresa.

A capacidade de êxito do administrador depende da sua competência de fazer com que o trabalho seja executado pelas pessoas que se reportam a ele. Se o gestor espera ter funcionários de qualidade ele precisa treinar e desenvolver o seu pessoal, do contrário passará a maior parte do seu tempo corrigindo erros e fazendo o trabalho ele mesmo. O treinamento, talvez, seja a mais negligenciada das funções administrativas e uma das mais importantes, nada substitui o treinamento. Uma pessoa pode ter inúmeras aptidões e experiência, mas ainda assim, precisa ser treinada nos moldes da empresa. É através do treinamento que o funcionário compreende os valores e os procedimentos relativos a uma determinada função.

A conceituação, segundo Marras (2007), diz que treinamento é um processo de assimilação cultural em curto prazo, que objetiva repassar ou reciclar conhecimentos, habilidades ou atitudes relacionados diretamente à execução de tarefas ou à sua otimização no trabalho.

Conforme Brandão; Guimarães (2001) em algumas organizações, o treinamento é um investimento empresarial destinado a capacitar uma equipe de trabalho a fim de reduzir ou eliminar a diferença entre $o$ atual desempenho e os objetivos propostos. O treinamento não deve ser considerado uma despesa, mas um investimento, cujo retorno é altamente compensador para a organização. $\mathrm{Na}$ instituição de saúde não será diferente, uma empresa em que a matéria prima é a vida humana não apenas precisa, mas também exige que seus profissionais sejam capacitados, treinados e habilitados para que o serviço oferecido à sua clientela seja de qualidade total.

O importante no treinamento é o surgimento de novidades que ocorrem na instituição. Para que sejam identificadas, deve-se partir de levantamentos e pesquisas internas capazes de localizálas, mas isto é um trabalho árduo e contínuo visando à percepção de variações no ambiente e necessidades futuras (CHIAVENATO, 2004).

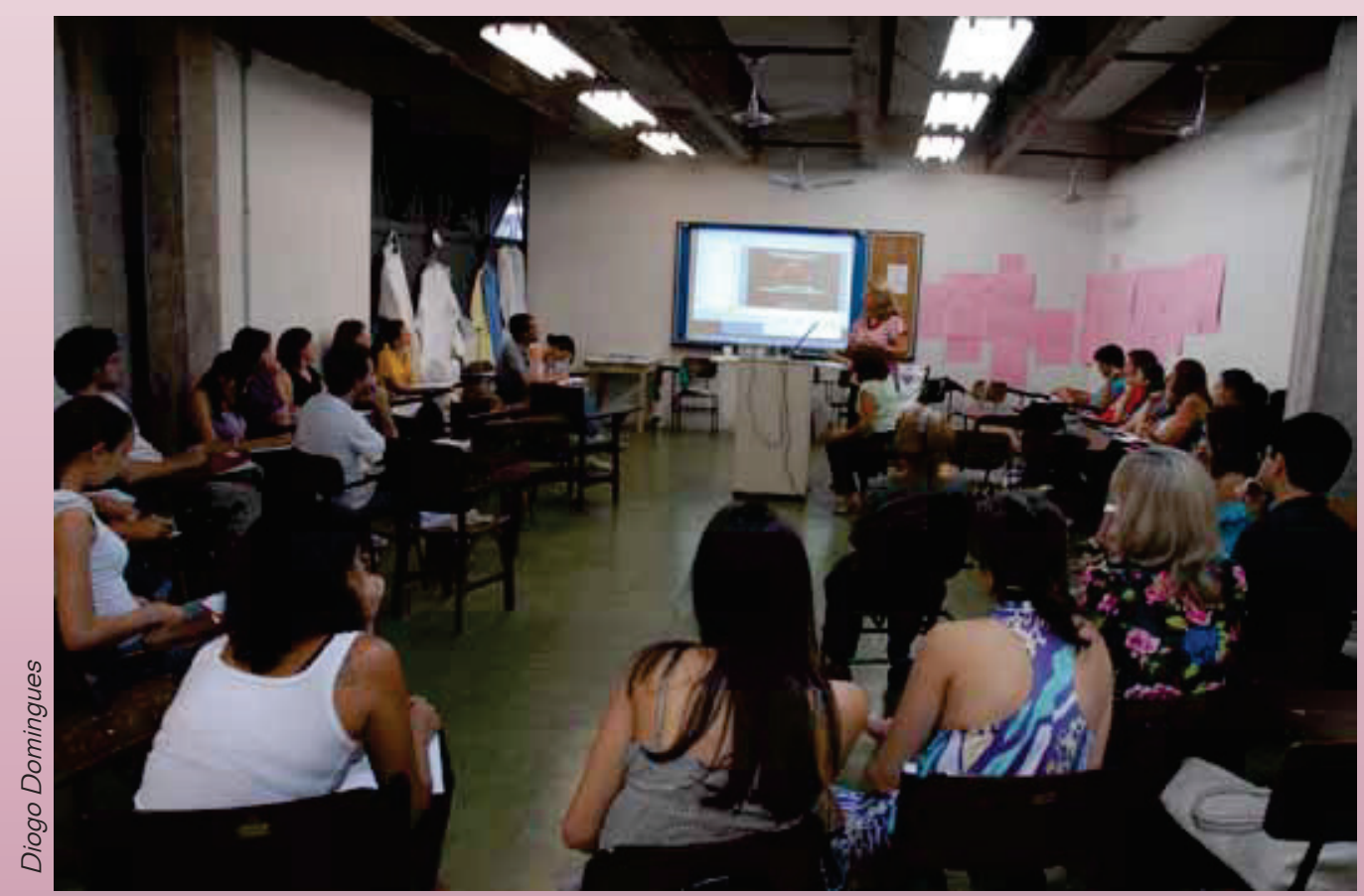




\title{
3.2. Funções e Objetivos do Treinamento e Desenvolvimento
}

Pio (2006, p. 1) conceitua desenvolvimento como:

\begin{abstract}
Um conjunto de atividades que objetiva explorar o potencial de aprendizagem e a capacidade produtiva das pessoas, visando mudanças de comportamentos e atitudes bem como a aquisição de novas habilidades e conhecimentos. Tem como meta maximizar o desempenho profissional e motivacional do ser humano, melhorando os resultados e consequentemente gerando um bom clima organizacional.
\end{abstract}

Após ser realizado o diagnóstico e serem localizadas as necessidades de treinamento é necessário estabelecer um programa integrado e coeso de acordo com as necessidades estratégicas da organização e imediatamente dar início aos trabalhos de treinar e capacitar as pessoas.

A definição de necessidades de treinamento, de acordo com Ferreira (2009), permite a construção de modelos e instrumentos de avaliação de necessidades e treinamentos os quais, quando operacionalizados, possibilitam: o estabelecimento de prioridades de treinamento, a formulação de sugestões de mudanças de desempenho, a descrição de objetivos educacionais, o fornecimento de bases sólidas para o desenho institucional, a execução e a avaliação de cursos, o encorajamento de trabalhares à participação na construção e na execução de ações educacionais, o alinhamento dos treinamentos oferecidos aos objetivos e às competências estratégicas da organização.

Portanto, uma das finalidades do treinamento é gozar da capacidade que o ser humano tem de aprender a adquirir novas habilidade e novos conhecimentos modificando comportamentos e atitudes (TACHIZAWA et al., 2001). Ampliar a performance profissional além de ser bom para os resultados da instituição servirá como fator de motivação para o funcionário.

Para os autores Tachizawa et al (2001), o treinamento tem como objetivo a preparação das pessoas para a execução imediata das diversas tarefas peculiares à organização; dando oportunidades para o contínuo desenvolvimento pessoal, não apenas no cargo atualmente ocupado, mas também em outros que o indivíduo possa vir a exercer; mudança nas atitudes das pessoas, a fim de criar entre elas um clima mais satisfatório, aumentando-lhes a motivação e tornando-as mais receptivas às técnicas de supervisão e gestão.

Treinar e desenvolver pessoas não são simplesmente informar, mas sim formar. Dar-lhes formação para aprenderem novas atitudes, ideias, conceitos e se tornarem mais eficazes naquilo que fazem.

\section{DIAGNÓSTICO DA NECESSIDADE DE TREINAMENTO E DESENVOLVIMENTO (T\&D)}

O levantamento das necessidades de treinamento (LNT) engloba a pesquisa e a respectiva análise pela qual se detecta o conjunto de carências cognitivas e inexperiências relativas ao trabalho existentes entre o conjunto de conhecimentos, habilidades e atitudes do indivíduo e as exigências do perfil do cargo (MARRAS, 2007).

Mas vale ressaltar que, segundo Menezes; Zerbini (2009), o processo de LNT consiste em uma tentativa sistematizada de identificação de problemas de desempenho que podem ser solucionados por meio de ações educacionais, e somente por estas.

O produto final de um serviço é sempre um sentimento. Os clientes ficam satisfeitos ou não conforme suas expectativas. Portanto, a qualidade do serviço é variável de acordo com o tipo de pessoa. É importante observar que os serviços possuem dois componentes de qualidade que devem ser considerados: o serviço propriamente dito e a forma como é percebido pelo cliente (LAS CASAS, 2008).

Conforme Brandão; Guimarães (2001) a total satisfação dos clientes é o que impulsiona a gestão pela qualidade, fator em torno do qual a organização deve agir em função. Afinal, os clientes são a própria razão de existência dela. Saber como eles avaliam os produtos ou serviços que thes são oferecidos é o primeiro passo.

Bohlander et al (2005) fornecem três formas de analisar rapidamente as necessidades de treinamento: análise da empresa através de exame de ambiente, estratégias e recursos da organização para determinar onde deve incidir a ênfase ao treinamento; análise da tarefa como processo de determinar que conteúdo de programa de treinamento devesse estar na base de um estudo das tarefas e deveres envolvidos no trabalho; análise das pessoas para determinação dos indivíduos específicos que precisam de treinamento. 
A pesquisa de necessidades deve abranger a organização, os indivíduos e o ambiente, devendo esta ser contínua e permanente. A partir da detecção de um problema, uma deficiência, deve-se planejar a pesquisa buscando suas possíveis causas.

Existem vários indicadores de necessidades de treinamento na organização, tais como substituições ou movimentação de pessoal, novos funcionários, licenças e férias do pessoal, modernização dos aparelhos, mudança de métodos e processos de trabalho, principalmente na saúde onde há novidades diárias trazidas pela evolução da ciência. Apesar dos indicadores, existem muitas formas de proceder no levantamento de tais necessidades (MAESTRO-FILHO, 2004).

Segundo Chiavenato (2004), estas são algumas técnicas para determinação das necessidades de treinamento dentro de uma empresa:

- Observações;

- Solicitações da gerência;

- Entrevistas;

- Conferências de grupos;

- Análises de cargos ou de atividades;

- Questionário de pesquisas;

- Testes ou exames;

- Avaliação de mérito ou de execução;

- Registros de pessoal;

- Relatórios da empresa ou de produção e

- Planejamento organizacional a longo prazo.

Não será fácil treinar e desenvolver habilidades, mas a organização precisa se dispor a enfrentar o desafio de oferecer conhecimento e oportunizar crescimento para sua equipe.

As principais causas do mau atendimento são déficits dos funcionários, sem nenhuma espécie de treinamento, atitudes negativas em relação aos clientes, ausência de filosofia de atendimento, e isso tudo só viabiliza ainda mais a necessidade de desenvolver competências.

\section{CONCLUSÃO}

A presente pesquisa sugere desenvolvimento de Recursos Humanos, atentando, não apenas para melhorar o desempenho dos funcionários em desenvolver tarefas, mas também para suas condições de crescimento pessoal e profissional, possibilitando que os funcionários tenham um desempenho mais dinâmico e de melhor qualidade.

Diante da problemática diagnosticada pôde-se concluir que é importante ter uma gestão comprometida com o treinamento dos seus funcionários. Não pode haver qualidade na prestação de serviços sem desenvolvimento e treinamento dos recursos humanos. 


\section{REFERÊNCIAS}

BOHLANDER, G; SNELL, S; SHERMAN, A. Administração de recursos humanos. São Paulo: Pioneira, 2005.

BRANDÃO, HP; GUIMARÃES, TA. Gestão de competências e gestão de desempenho: tecnologias distintas ou instrumentos de um mesmo construto? Revista de Administração de Empresas . Jan./ Mar. v. 41 . n. 1 . 2001.

CHIAVENATO, I. Gestão de Pessoas. 2. Ed. São Paulo; SP: Campus, 2004.

GIL, AC. Gestão de Pessoas, enfoque nos papéis profissionais. São Paulo, Atlas, 2006.

LACERDA, Érika Rodrigues Magalhães; ABBAD, Gardênia. Impacto do treinamento no trabalho: investigando variáveis motivacionais e organizacionais como suas preditoras. Rev. adm. contemp. 2003, vol. 7, n. 4, pp. 77-96.

LAS CASAS, AL. Qualidade total em serviços: conceitos, exercícios, casos práticos. 6 ed. São Paulo: Atlas, 2008.

MAESTRO-FILHO, AD. Modelo relacional entre modernização organizacional, praticas inovadoras de treinamento e satisfação no trabalho. Belo Horizonte. p. 281, 2004.

MARRAS, JP. Administração de Recursos Humanos: do operacional ao estratégico. $12^{\circ}$ edição. São Paulo, editora Futura, 2007.

MAXIMIANO, Antonio César Amaru. Introdução à administração. São Paulo: Atlas, 2000.

MENESES, PPM; ZERBINI, T. Levantamento de necessidades de treinamento: reflexões atuais. Análise, Porto Alegre, v. 20, n. 2, p. 50-64, jul./dez. 2009.

PIO, C. Treinamento e Desenvolvimento de pessoas: dois lados da mesma moeda. 2006.

TACHIZAWA, T; FERREIRA, VCP; FORTUNA, AAM. Gestão com Pessoas: uma abordagem aplicada às estratégias de negócios. São Paulo: FGV, 2001, cap. 9, 2ª . Ed. 